\title{
UTILIZATION OF CHESTNUT SHELL WASTES AS A DYEING AGENT FOR LEATHER INDUSTRY
}

\section{UTILIZAREA COJILOR DE CASTANE CA AGENT DE VOPSIRE PENTRU INDUSTRIA DE PIELĂRIE}

\author{
Ersin ONEM, Gokhan CIN, Ataman ALANKUS, Hasan PEHLIVAN, Mehmet Mete MUTLU*
}

Ege University, Faculty of Engineering, Department of Leather Engineering, 35100, Izmir, Turkiye, e-mail: deri@mail.ege.edu.tr

\author{
UTILIZATION OF CHESTNUT SHELL WASTES AS A DYEING AGENT FOR LEATHER INDUSTRY
}

ABSTRACT. In this study, extraction of dyestuff from chestnut shell wastes and dyeing properties were investigated. Firstly, feed/solvent (F/S): $1 / 10-1 / 20(\mathrm{~g} / \mathrm{ml})$, time: $1-2 \mathrm{~h}$, solvent: water, water $+\mathrm{NaOH}(2.5 \%, 5 \%, 10 \%)$ parameters at $90^{\circ} \mathrm{C}$ were examined for the extraction of chestnut shell. $43.61 \%$ extraction yield was obtained in water $+10 \% \mathrm{NaOH}, \mathrm{F} / \mathrm{S}: 1 / 10$ at $90^{\circ} \mathrm{C}$ for 1 hour as optimum parameters. Chestnut shell extract produced in optimum conditions was used as a dyeing agent in leather process. Potassium aluminium sulphate $\left[\mathrm{KAl}\left(\mathrm{SO}_{4}\right)_{2} \cdot 12 \mathrm{H}_{2} \mathrm{O}\right]$, copper sulphate $\left[\mathrm{CuSO} .5 \mathrm{H}_{2} \mathrm{O}\right]$ and Ferro sulphate $\left[\mathrm{FeSO} .7 \mathrm{H}_{2} \mathrm{O}\right]$ mordants were used after dyeing process to increase the fastness properties of leather. Rub, perspiration and light fastnesses were examined on the dyed leathers. Colour measurements of leathers were also carried out with Konica Minolta CM-3600d spectrophotometer. Mordants did not show significant effect on colour but fastness values varied by the used mordant types.

KEY WORDS: leather, dyeing, dyestuff, chestnut shell, valorisation, extract, waste

\section{UTILIZAREA COJILOR DE CASTANE CA AGENT DE VOPSIRE PENTRU INDUSTRIA DE PIELĂRIE}

REZUMAT. În acest studiu, s-a investigat extracția colorantului din coji de castane și proprietățile de colorare ale acestuia. Pentru a obține extractul din coji de castane, s-au examinat mai întâi parametri precum raportul substanță/solvent (F/S): 1/10-1/20 (g/ml), timpul: 1-2 h, solventul: apă, apă+NaOH $(2,5 \%, 5 \%, 10 \%)$ la $90^{\circ} \mathrm{C}$. S-a obținut un procent de extracție de $43,61 \%$ la parametrii optimi apă+NaOH, F/S: $1 / 10$ la $90^{\circ} \mathrm{C}$ timp de 1 oră. Extractul din coji de castane obținut în condiții optime a fost utilizat ca agent de vopsire la prelucrarea pieilor. După procesul de vopsire s-au utilizat mordanți precum sulfat de potasiu și aluminiu [KAl( $\left(\mathrm{SO}_{4}\right)_{2} \cdot 12 \mathrm{H}_{2} \mathrm{O}$ ], sulfat de cupru [CuSO $\left.\mathrm{Cu}_{4} \cdot 5 \mathrm{H}_{2} \mathrm{O}\right]$ și sulfat de fier $\left[\mathrm{FeSO}_{4} .7 \mathrm{H}_{2} \mathrm{O}\right]$ pentru a crește rezistența pieilor. S-a examinat rezistența la frecare, la transpirație și la lumină a pieilor vopsite. S-au efectuat măsurători de culoare cu spectrofotometrul Konica Minolta CM-3600d. Mordanții nu au avut un efect semnificativ asupra culorii, însă valorile rezistenței au variat în funcție de tipul de mordant utilizat.

CUVINTE CHEIE: piele, vopsire, colorant, coji de castane, valorificare, extract, deșeu

\section{L'UTILISATION DES COQUILLES DE CHÂTAIGNES COMME AGENT DE TEINTURE POUR L'INDUSTRIE DU CUIR}

RÉSUMÉ. Dans cette étude on a étudié l'extraction du colorant des coquilles de châtaignes et ses propriétés colorantes. Pour obtenir l'extrait de coquilles de châtaignes on a d'abord examiné des paramètres tels que le rapport substance/solvant (F/S): 1/10-1/20 ( $\mathrm{g} / \mathrm{ml}$ ), temps: $1-2$ heures, solvant: eau, eau+ $\mathrm{NaOH}(2,5 \%, 5 \%, 10 \%)$ à $90^{\circ} \mathrm{C}$. On a obtenu un taux d'extraction de $43,61 \%$ aux paramètres optimaux eau+ $\mathrm{NaOH}, \mathrm{F} / \mathrm{S}: 1 / 10$ à $90^{\circ} \mathrm{C}$ pendant 1 heure. L'extrait de coquilles de châtaignes obtenu dans des conditions optimales a été utilisé comme agent de coloration dans le traitement du cuir. Après la teinture, on a utilisé de mordants tel que le sulfate d'aluminium et de potassium [KAl $\left(\mathrm{SO}_{4}\right)_{2} \cdot 12 \mathrm{H}_{2} \mathrm{O}$ ], le sulfate de cuivre [CuSO $\left.{ }_{4} \cdot 5 \mathrm{H}_{2} \mathrm{O}\right]$, et le sulfate de fer $\left[\mathrm{FeSO}_{4} \cdot 7 \mathrm{H}_{2} \mathrm{O}\right]$ pour augmenter la résistance du cuir. On a examiné la résistance des cuirs teints à l'abrasion, à la transpiration et à la lumière. On a effectué des mesures de couleur avec le spectrophotomètre Konica Minolta CM-3600d. Les mordants n'ont pas eu d'effet significatif sur la couleur, mais les valeurs de résistance varient en fonction du type de mordant utilisé.

MOTS CLÉS: cuir, teinture, colorant, coquilles de châtaignes, récupération, extraction, déchet

\section{INTRODUCTION}

Chestnut is a kind of fruit cultivated in Asia, Europe and America continents of the Northern Hemisphere and in South America partially. Asia is the most important region and China is the leader of Asia. Southern Europe and Turkey (Mediterranean countries) are the second important regions [1]. According to FAO statistics [2], the world's largest producers of chestnut are China $(1,000,000$ tons), South Korea (75,000 tons), Italy and Turkey $(55,000$ tons), and Japan (26,000 tons).

Chestnut shells are the waste product from chestnut food processing and have not been fully utilised to date. The glace chestnut industry produces each year a huge amount of chestnut shells as waste [3]. The shell, which represents around $10 \%$ by weight of the chestnut, is removed in the peeling

\section{INTRODUCERE}

Castanele sunt o specie de fructe cultivate în Asia, Europa, America de Nord și parțial în America de Sud. Asia este cea mai importantă regiune, iar China este liderul Asiei. Europa de Sud și Turcia (țările mediteraneene) se situează pe locul doi ca importanță [1]. Potrivit statisticilor FAO [2], cei mai mari producători mondiali de castane sunt China (1.000.000 tone), Coreea de Sud (75.000 tone), Italia și Turcia (55.000 tone) și Japonia (26.000 tone).

Cojile de castane sunt deșeuri rezultate în urma prelucrării castanelor pentru consum și nu sunt în prezent utilizate la potențial maxim. Industria de castane confiate produce anual o cantitate enormă de coji de castane considerate deșeuri [3]. Coaja, reprezentând aproximativ $10 \%$ din greutatea castanei, este înlăturată în procesul de decojire și

"Correspondence to: Mehmet Mete MUTLU, Ege University, Faculty of Engineering, Department of Leather Engineering, 35100, Izmir, Turkiye, Tel.: +90 232311 2644, e-mail: mete.mutlu@ege.edu.tr 
process and used as fuel in the industry. These shells contain a large quantity of brown pigment which is mainly used in the food industry, where it is gradually replacing caramel pigment, with some toxicity at high concentrations [4]. Chestnut wood tannins are also of the most common hydrolyzable tannins used in leather industry as vegetable tanning agent [5].

Current arguments, environmental concerns, and related legal regulations have increased the demand for natural products in leather industry, similar to those of other branches. Research studies conducted in this context are becoming more crucial and valuable day by day to ensure environmental standards compliance. Research on utilization of $100 \%$ natural products as substitutes of chemical products widely used in the past are gradually gaining higher importance [6].

The prohibition of certain dyestuffs in the leather industry is also being contemplated as various regulatory frameworks worldwide are implemented. For example, the use of certain azo-dyes has been prohibited because they are difficult to biologically decompose and are known to constitute potential toxicity in living organisms [7]. The restrictions on the waste waters containing inorganic dyestuffs also impel the researchers to use natural dyestuffs. With the employment of various mordants, non-pollutant natural dyestuffs are convenient for obtaining a wide range of colours and good fastnesses [8]. In this study, optimum extraction yield of chestnut shell from the industrial wastes, dyeing of leather with the extractmordant combinations and fastness properties of the dyed leathers were researched.

\section{MATERIALS AND METHODS}

\section{Materials}

Chestnut shells as an industrial waste were supplied from a chestnut sweet producing food company in Turkey. Domestic wet-blue sheep skins were used as the leather material. Mordants of potassium aluminium sulphate [KAl $\left(\mathrm{SO}_{4}\right)_{2} \cdot 12 \mathrm{H}_{2} \mathrm{O}$ ], copper sulphate $\left[\mathrm{CuSO}_{4} \cdot 5 \mathrm{H}_{2} \mathrm{O}\right.$ ] and Ferro sulphate $\left[\mathrm{FeSO}_{4} \cdot 7 \mathrm{H}_{2} \mathrm{O}\right]$ were obtained from Borkim Chemicals (Turkey).

\section{Methods}

\section{Moisture Content of the Shells and Ash Content of the Extract}

Moisture content of the shells was determined according to SLC 113 standard method [9]. Ash contents of the extract were done to TS 4125 EN ISO utilizată în industrie pentru combustibil. Aceste coji conțin o mare cantitate de pigment maro, utilizat în principal în industria alimentară, unde înlocuiește treptat pigmentul pe bază de caramel, care este toxic în concentrații mari [4]. De asemenea, taninurile pe bază de lemn de castan se numără printre cele mai uzuale taninuri hidrolizabile utilizate în industria de pielărie ca agent tanant de origine vegetală [5].

Argumentele actuale, preocupările legate de mediu și reglementările locale au dus la creșterea cererii de produse naturale în industria de pielărie, precum și în alte ramuri ale industriei. Studiile efectuate în acest context capătă pe zi ce trece o importanță și o valoare din ce în ce mai mare, în vederea asigurării conformității cu standardele de mediu. Cercetările privind utilizarea unor produse 100\% naturale ca înlocuitori pentru produsele chimice utilizate pe scară largă în trecut capătă importanță din ce în ce mai mare [6].

Se are în vedere, de asemenea, interzicerea anumitor coloranți în industria de pielărie, pe măsură ce se pun în practică diverse cadre de reglementare la nivel mondial. De exemplu, utilizarea anumitor coloranți azoici a fost interzisă deoarece se descompun biologic foarte greu și sunt cunoscuți pentru toxicitatea potențială pentru organismele vii [7]. Restricțiile privind apele uzate cu conținut de coloranți anorganici îndeamnă cercetătorii să utilizeze coloranți naturali. Utilizați în combinație cu diferiți mordanți, coloranții naturali nepoluanți sunt convenabili pentru obținerea unei game largi de culori și unor rezistențe superioare [8]. În acest studiu, s-au investigat randamentul optim de extracție al cojilor de castane din deșeuri industriale, vopsirea pieilor cu combinații extract-mordant și proprietățile de rezistență ale pieilor vopsite.

\section{MATERIALE ȘI METODE}

\section{Materiale}

Cojile de castane ca deșeu industrial au fost furnizate de către o companie producătoare de dulciuri din Turcia. S-au utilizat piei de oaie tăbăcite în crom din surse locale. Mordanții utilizați, sulfat de potasiu și aluminiu [KAl $\left(\mathrm{SO}_{4}\right)_{2} \cdot 12 \mathrm{H}_{2} \mathrm{O}$ ], sulfat de cupru [CuSO $\left.\mathrm{CH}_{4} \cdot 5 \mathrm{H}_{2} \mathrm{O}\right]$ și sulfat de fier $\left[\mathrm{FeSO}_{4} .7 \mathrm{H}_{2} \mathrm{O}\right]$, au fost furnizați de Borkim Chemicals (Turcia).

\section{Metode}

Conținutul de umiditate al cojilor și conținutul de cenușă al extractului

Conținutul de umiditate al cojilor a fost determinat conform metodei standard SLC 113 [9]. Conținutul de cenușă al extractului a fost determinat conform standardului TS 4125 EN ISO 4047 [10]. Experimentele au fost efectuate în duplicat, făcându- 
4047 standard [10]. The experiments were done in duplicate and average contents were determined.

\section{Extraction of Chestnut Shells}

Chestnut shells were crushed and dried before the extraction processes. Extractions were performed by using distilled water as solvent. 10g ( \pm 0.05$)$ sample was used for each run. Temperature was kept constant at $90^{\circ} \mathrm{C}$ for the extractions and parameters of $\mathrm{F} / \mathrm{S}: 1 / 10-1 / 20 \mathrm{~g} / \mathrm{ml}$, time: $1-2 \mathrm{~h}$ and combination of water $+\mathrm{NaOH}$ were examined. Chestnut shell and water mixed at room temperature, heated and, once the selected temperature was attained, the alkali was added and contact time begun to run.

The extraction solutions were collected and filtered using a Whatman filter paper (no. 1) and then dried in an air oven at $90^{\circ} \mathrm{C}$. The extraction experiments were repeated two times.

\section{Extraction Yield}

$10 \mathrm{ml}$ of samples were taken from the extract solution into clean, dried and weighed glass dishes, in order to determine amount of carried solid matter into extraction medium. The extracts were dried in a hot-air oven $\left(100 \pm 2^{\circ} \mathrm{C}\right)$ until all the water evaporated and only the solid matter was left. The dishes were then cooled in a desiccator and weighed, in this way, the amount of solid matter was determined.

\section{Dyeing Process of Leather}

Process of wet-blue skins was given in Table 1. Skins were processed with the chestnut shell extract and then mordanted for dyeing. A group of leather samples were dyed with only extract without mordanting. se o medie a valorilor obținute.

\section{Obținerea extractului din coji de castane}

Cojile de castane au fost zdrobite și uscate înainte de procesele de extracție. Extracțiile au fost efectuate utilizând apă distilată ca solvent. S-au utilizat $10 \mathrm{~g}( \pm 0,05)$ de probă pentru fiecare test. Temperatura s-a menținut constantă la $90^{\circ} \mathrm{C}$ pentru extracții și s-au determinat parametrii F/S: 1/10-1/20 g/ml, timp: 1-2 h și combinația apă+NaOH. Cojile de castane și apa s-au amestecat la temperatura camerei, s-au încălzit și, odată ce s-a atins temperatura selectată, s-a adăugat baza și s-a măsurat timpul de contact.

S-au colectat și filtrat soluțiile de extracție utilizând hârtie de filtru Whatman (nr. 1), apoi s-au uscat în cuptor cu aer la $90^{\circ} \mathrm{C}$. Experimentele de extracție s-au realizat de două ori.

\section{Randament de extracție}

$\mathrm{S}$-au prelevat $10 \mathrm{ml}$ de probă din soluția de extract și s-au depus în vase de sticlă curate, uscate și cântărite, pentru a determina cantitatea de materie solidă în mediul de extracție. S-au uscat extractele într-un cuptor cu aer cald $\left(100 \pm 2^{\circ} \mathrm{C}\right)$ până ce s-a evaporat toată apa și a rămas doar materia solidă. Apoi vasele au fost răcite într-un exsicator și s-au cântărit, determinând astfel cantitatea de materie solidă.

\section{Procesul de vopsire a pielii}

Procesul de fabricare a pieilor cromate este prezentat în Tabelul 1. Pieile au fost prelucrate cu extract de coajă de castane și apoi tratate cu mordant pentru vopsire. $\mathrm{O}$ parte din probele de piele au fost vopsite doar cu extract, fără tratare cu mordant.

Table 1: Leather dyeing process

Tabelul 1: Procesul de vopsire a pielii

\begin{tabular}{|c|c|c|c|c|c|}
\hline $\begin{array}{l}\text { Process } \\
\text { Proces }\end{array}$ & $\%$ & $\begin{array}{l}\text { Substance } \\
\text { Substanță }\end{array}$ & $\begin{array}{l}\text { Temperature }\left({ }^{\circ} \mathrm{C}\right) \\
\text { Temperatură }\left({ }^{\circ} \mathrm{C}\right)\end{array}$ & $\begin{array}{l}\text { Time (min.) } \\
\text { Timp (min.) }\end{array}$ & $\begin{array}{l}\text { Remarks } \\
\text { Observaţii }\end{array}$ \\
\hline \multirow[t]{2}{*}{$\begin{array}{l}\text { Washing } \\
\text { Spălare }\end{array}$} & 100 & $\begin{array}{l}\text { Water } \\
\text { Apă }\end{array}$ & 30 & & \\
\hline & 0.5 & $\begin{array}{l}\text { Formic acid } \\
\text { Acid formic }\end{array}$ & & 45 & $\begin{array}{c}\text { Drain } \\
\text { Scurgere }\end{array}$ \\
\hline \multirow[t]{3}{*}{$\begin{array}{l}\text { Neutralisation } \\
\text { Neutralizare }\end{array}$} & 100 & $\begin{array}{l}\text { Water } \\
\text { Apă }\end{array}$ & 35 & & \\
\hline & 1 & $\begin{array}{l}\text { Sodium formate } \\
\text { Formiat de sodiu }\end{array}$ & & 10 & \\
\hline & 1 & $\begin{array}{l}\text { Sodium bicarbonate } \\
\text { Bicarbonat de sodiu }\end{array}$ & & $3 \times 15+45$ & $\begin{array}{c}\mathrm{pH}: 5.0-5.2 \text {, drain } \\
\mathrm{pH}: 5,0-5,2 \text {, scurgere }\end{array}$ \\
\hline $\begin{array}{l}\text { Washing } \\
\text { Spălare }\end{array}$ & 300 & $\begin{array}{l}\text { Water } \\
\text { Apă }\end{array}$ & 35 & 10 & $\begin{array}{c}\text { Drain } \\
\text { Scurgere }\end{array}$ \\
\hline $\begin{array}{l}\text { Dyeing-fatliquoring- } \\
\text { retanning } \\
\text { Vopsire-ungere- } \\
\text { retăbăcire }\end{array}$ & 100 & $\begin{array}{l}\text { Water } \\
\text { Apă }\end{array}$ & 40 & & \\
\hline
\end{tabular}




\begin{tabular}{|c|c|c|c|c|c|}
\hline & 5 & $\begin{array}{l}\text { Dyestuff } \\
\text { Vopsea }\end{array}$ & & 60 & \\
\hline & 1 & $\begin{array}{l}\text { Mordant* } \\
\text { Mordant* }\end{array}$ & & 45 & \\
\hline & 8 & $\begin{array}{l}\text { Combined natural and } \\
\text { synthetic fatliquor } \\
\text { Agent de ungere } \\
\text { combinat natural şi } \\
\text { sintetic }\end{array}$ & & 60 & \\
\hline & 2 & $\begin{array}{l}\text { Phenolic syntan } \\
\text { Sintan fenolic }\end{array}$ & & 20 & \\
\hline & 2 & $\begin{array}{l}\text { Synthetic tanning agent } \\
\text { Agent tanant sintetic }\end{array}$ & & 20 & \\
\hline & 1 & $\begin{array}{l}\text { Formic acid } \\
\text { Acid formic }\end{array}$ & & $3 \times 15+45$ & $\begin{array}{l}\text { Drain } \\
\text { Scurgere }\end{array}$ \\
\hline $\begin{array}{l}\text { Washing } \\
\text { Spălare }\end{array}$ & 300 & $\begin{array}{l}\text { Water } \\
\text { Apă }\end{array}$ & 20 & 10 & $\begin{array}{l}\text { Drain } \\
\text { Scurgere }\end{array}$ \\
\hline
\end{tabular}

\footnotetext{
* Potassium aluminium sulphate, copper sulphate and ferro sulphate.
}

\section{Colorimetric Measurements}

Konica Minolta CM-3600d brand spectrophotometer has been used for measuring the colours of the leather samples which were processed with plant extracts, and how each extract changes the leather colour has been examined compared to the control sample. The colour differences between the control sample and the leathers processed with plant extracts have been calculated according to CIE Lab-76 colour difference formula [11].

\section{Rub, Perspiration and Light Fastnesses}

Analyses of rub, perspiration and light fastness were carried out using standard procedures [12-14] and grey scale was used for the assessment of colour change.

\section{RESULTS AND DISCUSSION}

Average moisture content of the shell was determined as $13.15 \%$ before the extractions. Vázquez et al. reported an increase from 70 to $90^{\circ} \mathrm{C}$ implied an increase in the extraction yield more significant for chestnut shell [5]. Thus, temperature was kept constant at $90^{\circ} \mathrm{C}$ in our study, and effects of $\mathrm{NaOH}$ addition into water, $\mathrm{F} / \mathrm{S}$ ratio and time were investigated. Extraction yield results of chestnut shell were given in Table 2. de fier.

* Sulfat de potasiu și aluminiu, sulfat de cupru și sulfat

\section{Măsurători colorimetrice}

S-a utilizat spectrofotometrul Konica Minolta CM-3600d pentru măsurarea culorilor probelor de piele prelucrate cu extracte vegetale și s-a examinat modul în care fiecare extract modifică culoarea pielii în comparație cu proba martor. Diferențele de culoare dintre proba martor și probele de piele prelucrate cu extracte vegetale s-au calculat utilizând formula diferenței de culoare din recomandările CIE Lab-76 [11].

\section{Rezistența la frecare, transpirație și lumină}

Analizele de rezistență la frecare, transpirație și lumină s-au efectuat utilizând proceduri standard [12-14], utilizându-se scara de gri pentru a evalua modificarea culorii.

\section{REZULTATE ȘI DISCUṬII}

Conținutul mediu de umiditate al cojilor a fost determinat la 13,15\% înainte de extracție. Vázquez și colab. au raportat o creștere de la 70 la $90^{\circ} \mathrm{C}$, ceea ce presupune o creștere semnificativă a randamentului de extracție din cojile de castane [5]. Astfel, în studiul nostru, temperatura s-a menținut constantă la $90^{\circ} \mathrm{C}$ și s-au investigat efectele adăugării $\mathrm{NaOH}$ în apă, raportul F/S și timpul. Rezultatele randamentului de extracție din cojile de castane sunt prezentate în Tabelul 2. 
Table 2: Effect of the parameters on extraction yield

Tabelul 2: Influenţa parametrilor asupra randamentului de extracţie

\begin{tabular}{|c|c|c|c|c|c|}
\hline $\begin{array}{l}\text { Extraction parameters } \\
\text { Parametri de extracţie }\end{array}$ & $\begin{array}{l}\text { Temperature }\left({ }^{\circ} \mathrm{C}\right) \\
\text { Temperatură }\left({ }^{\circ} \mathrm{C}\right)\end{array}$ & $\begin{array}{l}\text { Solvent } \\
\text { Solvent }\end{array}$ & $\begin{array}{c}\text { Feed/ } \\
\text { Solvent } \\
\text { (g/ml) } \\
\text { Raport } \\
\text { substanţă/ } \\
\text { solvent (g/ } \\
\text { ml) } \\
\end{array}$ & $\begin{array}{l}\text { Time } \\
\text { (h) } \\
\text { Timp } \\
\text { (h) }\end{array}$ & $\begin{array}{c}\text { Extraction yield (\%) } \\
\text { Randament de extracţie (\%) }\end{array}$ \\
\hline EP1 & 90 & $\begin{array}{c}\text { Water } \\
\text { Apă }\end{array}$ & $1 / 20$ & 2 & 9.72 \\
\hline EP2 & 90 & $\begin{array}{l}\text { Water } \\
\text { Apă }\end{array}$ & $1 / 10$ & 2 & 11.79 \\
\hline EP3 & 90 & $\begin{array}{l}\text { Water } \\
\text { Apă }\end{array}$ & $1 / 10$ & 1 & 11.71 \\
\hline EP4 & 90 & $\begin{array}{c}\text { Water+2.5\% } \\
\mathrm{NaOH} \\
\text { Apă+2,5\% NaOH}\end{array}$ & $1 / 10$ & 1 & 30.16 \\
\hline EP5 & 90 & $\begin{array}{c}\text { Water+5\% NaOH } \\
\text { Apă+5\% NaOH }\end{array}$ & $1 / 10$ & 1 & 35.36 \\
\hline EP6 & 90 & $\begin{array}{l}\text { Water+10\% NaOH } \\
\text { Apă+10\% NaOH }\end{array}$ & $1 / 10$ & 1 & 43.61 \\
\hline
\end{tabular}

F/S: $1 / 10$ and $1 / 20$ ratios did not show significant differences (EP1, EP2). EP2 and EP3, time of 1 and 2 hour did not differ for the extraction yield. After reaching these results, parameters of $F / S: 1 / 10$ and time: $1 \mathrm{~h}$ were kept constant and effect of $\mathrm{NaOH}$ addition into water was examined. The maximum extraction yield from chestnut shell with the value of $43.61 \%$ was obtained in parameter of EP6. Addition of $10 \% \mathrm{NaOH}$ into water was increased the solubility and extraction yield was increased. After determination of maximum response, chestnut shell extracts were produced under the conditions of EP6 and collected extracts were used in dyeing process of the skins. Ash content of the extract produced in optimum conditions was found to be $26.27 \%$.

The colour values of dyed and mordanted leathers were analysed spectrophotometrically and provided in Table 3.
Rapoartele F/S: $1 / 10$ și $1 / 20$ nu au prezentat diferențe semnificative (EP1, EP2). EP2 și EP3, cu timpii de două ore, respectiv o oră nu au prezentat diferențe în ceea ce privește randamentul de extracție. După obținerea acestor rezultate, raportul F/S: 1/10 și timpul: $1 \mathrm{~h}$ s-au menținut constanți și s-a examinat influența adăugării $\mathrm{NaOH}$ în apă. Randamentul maxim de extracție din coji de castane cu valoarea de $43,61 \%$ a fost obținut în cazul experimentului EP6. Adăugarea a $10 \% \mathrm{NaOH}$ în apă a crescut solubilitatea și, prin urmare și randamentul de extracție. După determinarea răspunsului maxim, extractele de coji de castane au fost obținute în condițiile EP6, iar extractele colectate au fost utilizate în procesul de vopsire a pieilor. Conținutul de cenușă al extractului obținut în condiții optime a fost de $26,27 \%$.

Culoarea pieilor vopsite și tratate cu mordant a fost determinată spectrofotometric și valorile obținute sunt prezentate în Tabelul 3.

Table 3: $L, a, b$ values of dyed and mordanted leathers

Tabelul 3: Valorile $L, a, b$ ale pieilor vopsite şi tratate cu mordant

\begin{tabular}{|c|c|c|c|c|}
\hline $\begin{array}{l}\text { No } \\
\text { Nr. crt. }\end{array}$ & $\begin{array}{l}\text { Mordants } \\
\text { Mordant }\end{array}$ & $L$ & $a$ & $b$ \\
\hline 1 & $\begin{array}{l}\text { Dyestuff extract } \\
\text { Extract colorant }\end{array}$ & 39.25 & 10.74 & 18.16 \\
\hline 2 & $\begin{array}{l}\text { Extract }+ \text { Potassium aluminium sulphate } \\
\text { Extract }+ \text { Sulfat de potasiu şi aluminiu }\end{array}$ & 42.10 & 9.82 & 17.65 \\
\hline 3 & $\begin{array}{l}\text { Extract }+ \text { Copper sulphate } \\
\text { Extract }+ \text { Sulfat de cupru }\end{array}$ & 37.57 & 9.54 & 15.77 \\
\hline 4 & $\begin{array}{l}\text { Extract + Ferro sulphate } \\
\text { Extract + Sulfat de fier }\end{array}$ & 38.16 & 8.00 & 13.70 \\
\hline
\end{tabular}




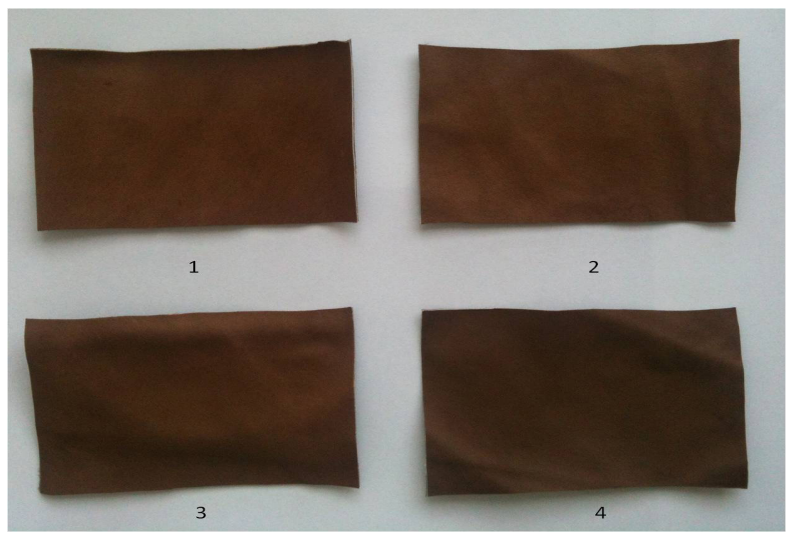

Figure 1. Appearance of dyed and mordanted leathers

Figura 1. Aspectul pieilor vopsite şi tratate cu mordant

It can be seen from the Figure 1 that mordants did not affect the colour of the leathers because chestnut shell extract gave dark brown colour. Potassium aluminium sulphate slightly increased the lightness of the leather with the $42.10 \mathrm{~L}$ value, and other mordants slightly decreased the $L$ values of the colours. Rub, perspiration and light fastnesses of the leathers dyed with chestnut shell extract and are seen in Tables 4-6.
Se poate observa din Figura 1 că mordanții nu au afectat culoarea pieilor, întrucât extractul din coji de castane a conferit o culoare maronie închisă. Sulfatul de potasiu și aluminiu a crescut ușor luminozitatea pielii, la valoarea $L$ de 42,10 , iar ceilalți mordanți au scăzut ușor valoarea $L$ a culorilor. Valorile rezistenței la frecare, la transpirație și la lumină a pieilor vopsite cu extract din coji de castane sunt prezentate în Tabelele 4-6.

Table 4: Rub fastnesses of dyed and mordanted leathers

Tabelul 4: Rezistenţa la frecare a pieilor vopsite şi tratate cu mordant

\begin{tabular}{|c|c|c|c|c|}
\hline \multirow{2}{*}{$\begin{array}{l}\text { Process } \\
\text { Proces }\end{array}$} & \multicolumn{2}{|c|}{$\begin{array}{l}\text { Leather } \\
\text { Piele - faţă }\end{array}$} & \multicolumn{2}{|c|}{$\begin{array}{c}\text { Felt } \\
\text { Piele - carne }\end{array}$} \\
\hline & $\begin{array}{l}\text { Dry rubbing } \\
\text { Frecare uscată }\end{array}$ & $\begin{array}{l}\text { Wet rubbing } \\
\text { Frecare umedă }\end{array}$ & $\begin{array}{l}\text { Dry rubbing } \\
\text { Frecare uscată }\end{array}$ & $\begin{array}{l}\text { Wet rubbing } \\
\text { Frecare umedă }\end{array}$ \\
\hline $\begin{array}{l}\text { Extract (Unmordanted) } \\
\text { Extract (fără mordant) }\end{array}$ & 4 & 4 & $4 / 5$ & 4 \\
\hline $\begin{array}{l}\text { Extract + Potassium aluminium suphate } \\
\text { Extract }+ \text { Sulfat de potasiu şi aluminiu }\end{array}$ & $4 / 5$ & $4 / 5$ & 5 & 5 \\
\hline $\begin{array}{l}\text { Extract }+ \text { Copper sulphate } \\
\text { Extract }+ \text { Sulfat de cupru }\end{array}$ & $4 / 5$ & $4 / 5$ & $4 / 5$ & $4 / 5$ \\
\hline $\begin{array}{l}\text { Extract + Ferro sulphate } \\
\text { Extract + Sulfat de fier }\end{array}$ & 5 & $4 / 5$ & 5 & 5 \\
\hline
\end{tabular}

Gray Scale for color change: 5: no change, 4: slight, 3: noticeable, 2: considerable, and 1: much.

Scara de gri pentru modificarea culorii: 5: nemodificat, 4: modificare uşoară, 3: modificare vizibilă, 2: modificare considerabilă şi 1: modificare pronunţată.

Table 5: Perspiration fastness on grain (G) and flesh (F) sides of leathers Tabelul 5: Rezistenţa la transpiraţie pe laturile de faţă şi de carne ale pielii

\begin{tabular}{|c|c|c|c|c|c|c|c|c|c|c|c|c|c|}
\hline \multirow{3}{*}{$\begin{array}{l}\text { Process } \\
\text { Proces }\end{array}$} & \multicolumn{2}{|c|}{$\begin{array}{c}\text { Color change } \\
\text { Modificarea culorii }\end{array}$} & \multicolumn{11}{|c|}{$\begin{array}{l}\text { Staining } \\
\text { Colorare }\end{array}$} \\
\hline & \multirow{2}{*}{$\begin{array}{l}\text { Grain } \\
\text { side } \\
\text { Faţă }\end{array}$} & \multirow{2}{*}{$\begin{array}{l}\text { Flesh } \\
\text { side } \\
\text { Carne }\end{array}$} & $\begin{array}{c}\text { Cellulose } \\
\text { acetate } \\
\text { Acetat de } \\
\text { celuloză }\end{array}$ & \multicolumn{2}{|c|}{$\begin{array}{l}\text { Cotton } \\
\text { Bumbac }\end{array}$} & \multicolumn{2}{|c|}{$\begin{array}{l}\text { Nylon } 6.6 \\
\text { Nylon } 6.6\end{array}$} & \multicolumn{2}{|c|}{$\begin{array}{l}\text { Polyester } \\
\text { Poliester }\end{array}$} & \multicolumn{2}{|c|}{$\begin{array}{l}\text { Acrylic } \\
\text { Acril }\end{array}$} & \multicolumn{2}{|c|}{$\begin{array}{l}\text { Wool } \\
\text { Lână }\end{array}$} \\
\hline & & & $\mathrm{G} \quad \mathrm{F}$ & G & $\mathrm{F}$ & G & $\mathrm{F}$ & G & $\mathrm{F}$ & G & $\mathrm{F}$ & G & $\mathrm{F}$ \\
\hline Extract & $4 / 5$ & $4 / 5$ & $4 / 5$ & 4 & $3 / 4$ & $3 / 4$ & $3 / 4$ & 4 & 4 & 4 & 4 & $4 / 5$ & 4 \\
\hline
\end{tabular}




\begin{tabular}{lcccccccccccccc} 
Extract+PAS & $4 / 5$ & $4 / 5$ & $4 / 5$ & 4 & 4 & $3 / 4$ & 4 & $3 / 4$ & $4 / 5$ & 4 & $4 / 5$ & 4 & $4 / 5$ & 4 \\
Extract+CS & 5 & $4 / 5$ & 5 & $4 / 5$ & 4 & $3 / 4$ & 4 & $3 / 4$ & $4 / 5$ & $4 / 5$ & $4 / 5$ & $4 / 5$ & $4 / 5$ & $4 / 5$ \\
Extract+FS & 5 & 5 & 5 & 5 & $4 / 5$ & $4 / 5$ & $4 / 5$ & $4 / 5$ & 5 & $4 / 5$ & 5 & 5 & 5 & $4 / 5$ \\
\hline
\end{tabular}

PAS: potassium aluminium sulphate, CS: copper sulphate, FS: ferro sulphate.
PAS: sulfat de potasiu și aluminiu, CS: sulfat de cupru, FS: sulfat de fier.

Table 6: Light fastness values of dyed leathers

Tabelul 6: Valorile rezistenței la lumină a pieilor vopsite

\begin{tabular}{|c|c|}
\hline $\begin{array}{c}\text { Process } \\
\text { Proces }\end{array}$ & $\begin{array}{c}\text { Light fastness } \\
\text { Rezistenţa la lumină }\end{array}$ \\
\hline Extract & 3 \\
\hline Extract+PAS & $3 / 4$ \\
\hline Extract + CS & $3 / 4$ \\
\hline Extract+FS & 4 \\
\hline
\end{tabular}

Although mordant applications did not affect the colour of the leathers, they increased the fastness values compared to unmordanted leathers. The best rub fastness was obtained with ferro sulphate mordant and dry rubbing of the leathers was relatively better than wet rubbing. Perspiration fastnesses on the grain and flesh sides of leathers did not considerably differ, but ferro sulphate gave the best fastness values again for both perspiration and light fastness. Onem et al. also reported that the highest rub, perspiration and light fastnesses of the leathers dyed with Rubia tinctorum extract were obtained with FS mordant [8]. Ozgunay determined the light fastness of chestnut tannin as hydrolysable tannin better than condensed tannins [15]. Light fastness of chestnut shell extract was found as 3 in this study and increased to 4 with FS mordant. Onem et al. researched the dyeing properties of the extract from onion skins (Allium cepa) as a natural dyeing agent [16]. Chestnut shell extract showed better light fastness properties than Allium cepa on leather. For textile industry, Wang et al. [4] purposed to develop an environmentally friendly dyeing process using brown pigment from chestnut shells obtained from foodstuff residues. The brown pigment was extracted from the raw material and purified with solvents containing EtOH. It was then used to dye flax fabric in aqueous solution with added $\mathrm{NaCl}$ as a dye accelerator. The effects of dyeing conditions and fastness were investigated. The dyed fabrics showed lower fastness to washing and higher fastness to rubbing and light.

\section{CONCLUSIONS}

In this study, a waste product, chestnut shells from food industry, was converted to a usable product with an economic value, a natural dyestuff used
Deși utilizarea mordanților nu a afectat culoarea pieilor, aceștia au dus la creșterea valorilor de rezistență în comparație cu pieile netratate cu mordant. Cea mai bună rezistență la frecare a fost obținută la utilizarea mordantului pe bază de sulfat de fier, iar rezistența la frecare uscată a fost relativ mai bună decât rezistența la frecare umedă. Rezistența la transpirație pe partea de față și pe partea de carne nu a prezentat nicio diferență semnificativă, însă utilizarea sulfatului de fier a avut ca rezultat cele mai bune valori atât pentru rezistența la transpirație, cât și pentru rezistența la lumină. Onem și colab. au raportat, de asemenea, că cele mai mari valori ale rezistenței la frecare, la transpirație și la lumină a pieilor vopsite cu extract de Rubia tinctorum au fost obținute cu mordant pe bază de sulfat de fier [8]. Ozgunay a constatat că rezistența la lumină a pieilor tratate cu tanin hidrolizabil pe bază de castane este mai bună decât cea a pieilor tratate cu taninuri condensate [15]. Rezistența la lumină a pieilor tratate cu extract din coji de castane a avut valoarea 3 în acest studiu și a crescut până la 4 la utilizarea mordantului pe bază de sulfat de fier. Onem și colab. au studiat proprietățile de vopsire ale extractului din coji de ceapă (Allium сера) ca agent de vopsire natural [16]. Pielea tratată cu extractul din coji de castane a prezentat proprietăți de rezistență la lumină mai bune decât cea tratată cu Allium cepa. În industria textilă, Wang și colab. [4] au dezvoltat un proces de vopsire ecologic utilizând pigment maro din coji de castane obținute din deșeuri din industria alimentară. Pigmentul maro a fost extras din materia primă și s-a purificat cu solvenți cu conținut de EtOH. S-a utilizat apoi la vopsirea țesăturii de in în soluție apoasă cu adaos de $\mathrm{NaCl}$ ca accelerator de vopsire. S-au investigat efectele condițiilor de vopsire și rezistența. Tesăturile vopsite au prezentat valori mai scăzute ale rezistenței la spălare și valori crescute ale rezistenței la frecare și lumină.

\section{CONCLUZII}

În acest studiu, s-a convertit un deșeu, cojile de castane din industria alimentară, într-un produs 
in dyeing of leather. 43.61\% extract was obtained after optimization studies in water $+10 \% \mathrm{NaOH}, \mathrm{F} / \mathrm{S}$ : $1 / 10$ at $90^{\circ} \mathrm{C}$ for 1 hour. The chestnut dyestuff gave dark brown colour with $L=39.25, a=10.74, b=18.16$ values and the colour did not change much with mordant application. However the rub fastness, perspiration fastness and light fastness properties of leathers dyed with chestnut shell extract enhanced with the mordant usage. The dyeing properties of leathers were found acceptable and can be enhanced with different mordants and dyeing techniques. Considering that, in recent years there is a demand on usage of ecologic and natural products in the world, usage of environmental friendly vegetable dyeing agents and producing natural leathers and colours can be more advantageous to gain the admiration and attention of consumers. utilizabil cu valoare economică, și anume un colorant natural utilizat la vopsirea pieilor. S-a obținut, după studii de optimizare, $43,61 \%$ extract în apă $+10 \% \mathrm{NaOH}$, F/S: $1 / 10$ la $90^{\circ} \mathrm{C}$ timp de 1 oră. Colorantul pe bază de coji de castane a conferit o culoare maronie închisă cu valorile $L=39,25, a=10,74, b=18,16$, iar culoarea nu s-a modificat vizibil la utilizarea mordanților. Pe de altă parte, proprietățile de rezistență la frecare, la transpirație și la lumină ale pieilor vopsite cu extract din coji de castane s-au îmbunătățit în cazul utilizării mordanților. Proprietățile de vopsire ale pieilor obținute au fost considerate acceptabile și pot fi îmbunătățire cu diferiți mordanți și tehnici de vopsire. Având în vedere că în ultimii ani există o mare cerere la nivel global de utilizare a produselor ecologice și naturale, utilizarea agenților de vopsire ecologici pe bază de plante și fabricarea pieilor naturale cu coloranți naturali poate fi mai avantajoasă în ceea ce privește atragerea admirației și atenției consumatorilor.

\section{REFERENCES}

1. Pereira-Lorenzo, S., Ramos-Cabrer, A.M., Diaz-Hernandez, M.B., Ciordia-Ara, M., Rios-Mesa, D., Chemical composition of chestnut cultivars from Spain, Sci Hortic-Amsterdam, 2006, 107, 3, 306-314.

2. FAO: available at: http://faosat.fao.org/default.aspx, last access: January, 2014.

3. Gómez, J., Pazos, M., Couto, S.R., Sanromán, M., Chestnut shell and barley bran as potential substrates for laccase production by Coriolopsis rigida under solid-state conditions, J Food Eng, 2005, 68, 3, 315-319.

4. Wang, L., Li, J., Feng, H., Dyeing of flax fabric with natural dye from chestnut shells, Pigm Resin Technol, 2009, $38,6,347-352$.

5. Vázquez, G., González-Alvarez, J., Santos, J., Freire, M.S., Antorrena, G., Evaluation of potential applications for chestnut (Castanea sativa) shell and eucalyptus (Eucalyptus globulus) bark extracts, Ind Crop Prod, 2009, 29, 2-3, 364-370.

6. Adiguzel Zengin, C.A., Crudu, M., Maier, S.S., Deselnicu, V., Albu, L., Gulumser, G., Bitlisli, B.O., Basaran, B., Mutlu, M.M., Eco-leather: Chromium-free leather production using titanium, oligomeric melamine-formaldehyde resin, and resorcinol tanning agents and the properties of the resulting Leathers, Ekoloji, 2012, 21, 82, 17-25.

7. Reife, A., Freeman, S., Environmental chemistry of dyes and pigments, John Wiley\&Sons Inc., New York, 1996.

8. Onem, E., Gulumser, G., Ocak, B., Evaluation of natural dyeing of leather with Rubia tinctorum extract, Ekoloji, 2011, 20, 80, 81-87.

9. Official Methods of Analysis, SLC-113, Determination of moisture, Society of Leather Technologists and Chemists, 1996.

10. TS 4125 EN ISO 4047, Leather - Determination of sulphated total ash and sulphated water - Insoluble ash. Turkish Standards Institution, Ankara, 2000.

11. CIE (Comission Interantionalle DeL'Eclairage), Official recommendations on uniform colour spaces colour difference equations metric colour terms, 1976.

12. TS EN ISO 11640, Leather - Tests for colour fastness - Colour fastness to cycles of to-and-fro rubbing. Turkish Standards Institution, Ankara, 2013.

13. TS EN ISO 11641, Leather - Tests for colour fastness - Colour fastness to perspiration. Turkish Standards Institution, Ankara, 2013.

14. International standard ISO 105-B02:2000/Amd.2.2000(E), 2000, Color fastness to artificial light: Xenon arc fading lamp test.

15. Ozgunay, H., Lightfastness properties of leathers tanned with various vegetable tannins, J Am Leather Chem As, 2008, 103, 10, 345-351.

16. Onem, E., Mutlu, M.M., Gunay, S., Azeri, H., Natural dyestuff extraction from onion (Allium cepa) skin and utilization for leather dyeing, Tekstil ve Mühendis, 2012, 19, 88, 1-8.

Article received/Data primirii articolului: 19.08.2016

Accepted/Acceptat la data: 28.10.2016 